\title{
Filter design masks for C37.118.1a-compliant frequency-tracking and fixed-filter M-class Phasor Measurement Units (PMUs)
}

\author{
Andrew J. Roscoe \\ Department of Electronic and Electrical Engineering \\ University of Strathclyde \\ Glasgow, UK \\ Andrew.J.Roscoe@strath.ac.uk
}

\author{
Bill Dickerson \\ Arbiter Systems \\ Paso Robles, CA, USA \\ Kenneth E. Martin \\ Electric Power Group \\ Portland, OR, USA
}

\begin{abstract}
The new amendment to the Phasor Measurement Unit (PMU) standard C37.118.1a makes several significant changes, compared to the standard C37.118.1 (2011). This paper highlights some of the most important changes, with a particular emphasis applied to how those changes relate to the way that an M-class PMU filter needs to be designed. In particular, there is a delicate trade-off between passband flatness (the bandwidth test) and stopband rejection in the Out-Of-Band (OOB) test. For a PMU algorithm using frequency-tracking and adaptive filters, it is shown that passband flatness can be relaxed to about $2.5 \mathrm{~dB}$, but that the stopband needs to begin up to $14.8 \%$ closer to $0 \mathrm{~Hz}$ than for a fixed-filter PMU. This is partly due to the exact procedures of the C37.118.1a "OOB" testing, and partly due to the adaptive nature of a frequency-tracking PMU filter section. Both the above lead to modified filter masks being required for frequency-tracking devices, compared to the mask required for fixed-filter devices. The M-class PMU with reporting rate $25 \mathrm{~Hz}$ is the most difficult to design, for reasons given in this paper. The validity of the masks is shown using filter bode plots and simulated C37.118.1a test results of a fixed-filter and frequencytracking device which have been designed to meet the masks defined in this paper.
\end{abstract}

Keywords-Power system measurements, Fourier transforms, Frequency measurement, Power system faults, Phase estimation, Power system state estimation, Power system parameter estimation

\section{TABLE OF NOMENCLATURE}

$A \quad$ Amplitude of interharmonic (pu)

$F(f) \quad$ Baseband filter response (Phasor-path filter)

$F E \quad$ Frequency error $(\mathrm{Hz})$

$F_{F}(f) \quad$ Baseband filter response (Frequency-path filter)

$F_{\text {ROCOF }}(f)$ Baseband filter response (ROCOF-path filter)

$F_{r} \quad$ Bandwidth required

$F_{S} \quad$ Reporting Rate $(\mathrm{Hz})$

$f \quad$ Fundamental frequency $(\mathrm{Hz})$

$f_{0} \quad$ Nominal frequency $(\mathrm{Hz})$

$f_{I H} \quad$ Interharmonic frequency $(\mathrm{Hz})$

$\begin{array}{cl}f_{M} & \text { Modulation frequency }(\mathrm{Hz}) \\ f_{T} & \text { Tuned frequency for a tracking PMU }(\mathrm{Hz}) \\ H & \text { Per-unit inertia (s) } \\ M & \text { Modulation depth }(\mathrm{pu}) \\ R F E & \text { ROCOF error (Hz/s) } \\ R O C O F & \text { Rate of change of frequency } \\ V & \text { Phasor (pu voltage) } \\ V_{\text {Meas }} & \text { Measured Phasor (pu voltage) } \\ \Delta V_{\text {Meas }} & \text { Measured Phasor heterodyned to nominally } \\ & (1+0 \mathrm{j}) \text { to analyse OOB disturbance effects (pu } \\ & \text { voltage) } \\ V_{\text {Error }} & \text { Measured Phasor error (pu voltage) }\end{array}$

\section{INTRODUCTION}

The present standard IEEE C37.118.1 was issued in 2011 [1] and presented a significant change relative to the 2005 standard. Specifically, dynamic requirements such as bandwidth, response time, delay time, latency, and frequency ramps were added. These are tested by applying amplitude and phase modulated signals, amplitude and phase steps, and frequency ramps. However, between 2011 and 2013, some issues were identified with the C37.118.1 (2011) standard. These are addressed with a new amendment C37.118.1a. In section III, some observations concerning RFE (Rate of change of Frequency Error) requirements/limits in C37.118.1a are made, from an application standpoint

However, the main focus of this paper is on the updated requirements for the bandwidth and Out-Of-Band interference (OOB) tests, and the implications for M-class Phasor Measurement Unit (PMU) design. The exact way these tests are carried out, and their stated limits, defines the way that filtering must be designed and implemented. In particular, it is shown in this paper that the required filter masks are different for fixed-filter and frequency-tracking devices. 
In both fixed-filter and frequency-tracking devices, a quadrature oscillator is used to decode the phasor components from the AC signal using a heterodyne process. The fixed filter design keeps the quadrature oscillator at the nominal system frequency, $f_{0}$ where the frequency tracking filter adjusts the quadrature oscillator to the actual system frequency $f$. While a viable mask for fixed-filter devices is given in [2], a frequencytracking PMU with adaptive filtering requires a different filter mask to comply with the standard. Equations developed in this paper allow such masks to be created.

The two tests: bandwidth and OOB, are the focus of this paper because between them they define the required filter response shape in the frequency domain. The bandwidth test is designed to test the passband response of the filtering, at frequencies close to the fundamental. If the attenuation of the filter is too high anywhere within the passband then the PMU will fail this test. The OOB test will fail if the filter attenuation is not large enough in the stop-band, at frequencies away from the fundamental. These two tests therefore define the overall requirement for filter mask - a lower gain limit in the passband and an upper gain limit in the stopband. By contrast, the steady-state tests are much more a test of calibration and timing accuracy, and signal-to-noise ratio within the PMU front-end signal conditioning and sampling. Even the harmonic test, as it stands, will pass virtually by default if the masks designed in this paper are complied with. This is because the filter attenuation at harmonic frequencies will be far in excess of the values in the close-in-stopband due to rolloff, while the Frequency Error (FE) requirements for the harmonics test are only halved $(0.005 \mathrm{~Hz})$ relative to the requirement for the $\mathrm{OOB}$ test $(0.01 \mathrm{~Hz})$. If the harmonics test is adjusted in future to include finite/useful RFE limits, such as those described in section III, then there would be additional requirements on filter masks to have deep notches at heterodyned frequencies where harmonics fall.

The dynamic step tests have a big impact on PMU filter design, but they do not constrain or define the frequencydomain filter masks. The dynamic step tests restrict the maximum time width of the window and the proportion of negative-weighted samples allowed (affecting overshoot). Shorter windows and restrictions on overshoot make it harder to achieve the required combinations of filter flatness, fast cutoff, and stopband attenuation. They affect the actual but not the required filter performance. So, while dynamic step test requirements affect filter design, they do not define the filter mask - they just restrict the design options available to achieve the masks. Frequency ramp and dynamic step tests will fail if PMU timestamping and any related calibration corrections are not correctly applied, but again this is a separate matter from the required filter mask shape in the frequency domain.

\section{THE REFERENCE ALGORITHM, RFE TEST LIMITS, AND APPLICATION REQUIREMENTS}

A "Reference" PMU algorithm design is presented within appendix $\mathrm{C}$ of $\mathrm{C} 37.118 .1$ [1], and appendix $\mathrm{C}$ of $\mathrm{C} 37.118 .1 \mathrm{a}$ [2], including block diagrams. It is important to note that the standard states "It is given for information purposes only, and does not imply being the only (or recommended) method for estimating synchrophasors. Its purpose is to establish common ground for understanding performance requirements and confirming their achievability". The reference model achieves what it states above, but is not used in any commercial PMUs to the authors' knowledge. Presently, C37.118.1a contains a series of tests with limits that can be complied with by the major PMU manufacturers, and also the Reference algorithm. This has required a certain degree of compromise in some areas. The tests and limits reflect performance achievable by both the Reference algorithm and all of the present major manufacturer's devices. The tests and limits do not necessarily reflect present or future application requirements, partly because these are not yet well quantified by the industry.

In particular, the steady-state tests in C37.118.1a have suspended limits for RFE for M-Class devices with harmonics added, and harmonics testing is only carried out with fundamental frequency $f$ fixed at the nominal frequency $f_{0}$. This means that C37.118.1a compliance provides little information as to the likely ROCOF (Rate of Change of Frequency) error (RFE) within a real-world network where frequency may be off-nominal and harmonics may be present. This may pose a problem for PMU users who wish to apply PMUs within schemes such as anti-islanding detection, where ROCOF trip settings were historically in the regions of $0.125-0.2 \mathrm{~Hz} / \mathrm{s}$ in the UK [3]. Alternatively, PMU outputs might be used to stabilise a network by despatching "inertia" from storage via the equation:

$$
\Delta P_{p u}=-\frac{2 H}{f_{0}} \frac{d f}{d t}
$$

where $f_{0}$ is nominal frequency, $H$ is the per-unit inertia (in seconds) of a device, $d f / d t$ is the measured ROCOF, and $\Delta P_{p u}$ is the required power adjustment to the storage device, as a proportion of rated power. If $H$ is set to be a useful value such as $H=6$ to provide network support, then by (1) a $0.4 \mathrm{~Hz} / \mathrm{s}$ RFE would lead to a $9.6 \% \Delta P_{p u}$ error which is significant for a multi-MW device. If the RFE is oscillating or noisy, as it most likely is under a harmonic or interharmonic interference, or offnominal operation, this represents a violent, high frequency, and possibly damaging input to the device controls. Additional filtering would need to be added. This itself is problematic since inertial response needs to be rapid; within $500 \mathrm{~ms}$ or less [3].

Existing and future usage scenarios for PMUs will include their placement at locations contaminated by finite levels of (perhaps unbalanced) harmonics and switching interharmonics from converter-connected renewables and HVDC (HighVoltage DC) links, at frequencies which are rarely exactly nominal. An M-class PMU with a response time and window length of about $200-400 \mathrm{~ms}$ offers the possibility of making measurements with $\mathrm{RFE}<<0.1 \mathrm{~Hz} / \mathrm{s}$ under off-nominal frequency conditions containing such interferences [4] [5]. At present, C37.118.1a itself does not provide a formal verification of this, and some PMUs (including the Reference algorithm) may provide quite poor RFE in these scenarios. However, many actual PMUs can make these RFE measurements very well, using fixed-filter or frequencytracking algorithms. A useful feature of subsequent standard development could be to quantify the actual application

This is a postprint of a paper submitted to and accepted for publication in IEEE Transactions on Instrumentation and Measurement [http://dx.doi.org/10.1109/TIM.2015.2445111] and is subject to IEEE copyright. 
scenarios and requirements for RFE measurement, and marry these with achievable results, so that useful and achievable tests and limits could be applied.

If the standard changes in future, then the required mask filter shapes (to achieve C37.118.1a compliance) given in this paper may also change. In particular, introduction of some realistically useful RFE requirements during the harmonics test and OOB tests would require additional deep notches at heterodyned frequencies where harmonics fall. However, the standard C37.118.1a is currently in force, and so the remainder of this paper uses the exact $\mathrm{C} 37.118 .1 \mathrm{a}(2014)$ requirement as the basis for the mask designs. Nevertheless, any user of a PMU within a particular application, solution or environment, will need to make their own assessment of whether C37.118.1a compliance guarantees acceptable accuracy or response/latency. Different network control/protection functions will have different priorities of accuracy, passband bandwidth, rejection of unwanted components, and response time. In some applications, the optimal solution may be a device which is not $\mathrm{C} 37.118 .1 \mathrm{a}$-compliant but that provides a tailored solution to that application.

\section{CANDIDATE ALGORITHM DESIGNS}

The aim of this paper is to describe the filter mask shapes which are required to conform to $\mathrm{C} 37.118 .1 \mathrm{a}$ by using a broadband rejection of stopband frequencies to guarantee OOB compliance, as well as providing the required passband flatness. This paper is most relevant to PMUs which use either fixed filters, or frequency-tracking PMUs whose heterodyne stage tracks the fundamental frequency. In these frequencytracking devices, it is assumed that the filter/window shape remains constant, but that the time length can be dilated or contracted proportionately to fundamental frequency, so that spectral leakage is reduced, by adaptively moving any filter zeros which are placed to reject harmonics.

Many of the presented PMU algorithms in academic literature avoid the issue of the OOB test, and present algorithms using windows which are too short to provide sufficient broadband stopband attenuation to pass the OOB test in C37.118.1 or C37.118.1a. For example, "Least Squares" [6] or "Taylor Fourier Transform" (TFT) [7] [8], and "Interpolated DFT (iDFT)" [9] [10] approaches have all recently been proposed. These algorithms all use fixed-filter approaches and generally suffer from degraded performance as frequency deviates from nominal, though iDFT methods aim to significantly reduce the degradation over a limited frequency range. The windows presented in those works are: 2-4 cycle Maximum Sidelobe Decay (MSD) (Hann) and Minimum Sidelobe Level (MSL) windows [6], rectangular 4 cycle window [7], 3-cycle Kaiser window [8], 2-3 cycle MSD \& MSL windows [9], 2-5 cycle Hann window [10].

Another fixed-filter TFT device is presented in [11]. By contrast to the above devices, a $50 \mathrm{~Hz}$ reporting-rate device is demonstrated with a long enough filter length (5.8 cycle Kaiser window) to comply with $\mathrm{C} 37.118 .1 \mathrm{OOB}$ tests, which are also performed. The window length would need to be extended from $\sim 6$ cycles to $\sim 10$ cycles to comply with C37.118.1a for a $50 \mathrm{~Hz}$ reporting device .
The "C37.118.1a Reference" algorithm [1] [2] is the most well-known fixed-filter PMU algorithm. M-class compliance for reporting-rates $50 / 60 \mathrm{~Hz}$ requires filter lengths of the order of 9-10 cycles [1] [2]. Slower reporting rates require longer filters.

Some algorithms use frequency-tracking, so that the algorithm tunes itself to constantly track the moving fundamental [4] [5] [12] [13] [14]. The example frequencytracking PMU used later in sections VII \& VIII is derived from the design given in [4] [5] [15], with Fig. 1 in [5] showing a representative detailed block diagram and the presentation [16] showing further details. Frequency-tracking minimises errors when frequency is off-nominal, potentially over a wide range, by keeping the fundamental at the centre of the passband. If the filters are carefully designed, the filter notches (zeros) can also be moved to track harmonics. The example [4] [5] represents a PMU which has these characteristics, which is extended in [15] [16] to have window lengths and shapes appropriate to achieve C37.118.1(a) compliance.

Some PMU algorithms attempt to use shorter windows to provide OOB-rejection capability by detecting interharmonics as they appear in real-time and inserting specific notches to reject just those individual frequency components. Such algorithms include the the Kalman filter technique [13] [14] and the compressive sensing technique [17] which uses a 5 cycle window for $50 \mathrm{~Hz}$ reporting. These PMUs may be able to pass the OOB test when single interharmonics only are applied, but may not produce good results in the presence of more broadband flicker, i.e. near an arc furnace or when a modulated "ripple-control" signal is being injected into the network. For such algorithms, the frequency-domain performance change is much more than a dilation or contraction proportional to fundamental frequency. The change is much more radical due to real-time dynamic signal conditions, and therefore derivation of an appropriate filter mask to achieve C37.118.1a compliance is complex, and beyond the scope of this paper.

Most proposed algorithms use symmetric FIR (Finite Impulse Response) windows since these most easily meet the C37.118.1a response and delay times. However, [13] [14] is notably different since it proposes the use of Kalman filtering which is essentially an IIR (Infinite Impulse Response) technique.

Many modern industrial PMUs e.g. [18] (whose algorithms are generally more conventional than the academic references above) can already actually perform significantly better than the C37.118.1 a limits in many of the tests, by using appropriate window function shapes and lengths, and by using suitable hardware.

\section{M-CLASS FILTER FLATNESS AND BANDWIDTH}

The bandwidth test is changed under C37.118.1a. Previously there were two tests: one with amplitude modulation only, and one with amplitude and phase modulation coincidentally. The combination of amplitude and phase modulation in C37.118.1 made it difficult to identify problems due to signal processing in this test, as the effects could sometimes occur destructively or constructively, and in complex non-linear manners [19]. So, the amplitude and phase

This is a postprint of a paper submitted to and accepted for publication in IEEE Transactions on Instrumentation and Measurement [http://dx.doi.org/10.1109/TIM.2015.2445111] and is subject to IEEE copyright. 
modulations are now applied separately in two sequential tests: with $0.1 \mathrm{pu}$ amplitude and then $0.1 \mathrm{rad}$ modulation applied. The required bandwidth of the PMU is set by a value $F_{r}$, calculated as $F_{r}=F_{S} / 5 \quad\left(F_{S}=\right.$ reporting rate), but limited to a maximum value of $5 \mathrm{~Hz}$.

The maximum allowable TVE (Total Vector Error) during this test is set at 3\% and it is interesting to analyse what this actually means. When the $0.1 \mathrm{pu}$ amplitude or $0.1 \mathrm{rad}$ phase modulation is applied to a $1 \mathrm{pu}$ fundamental at nominal frequency $f_{0}$, the true phasor should oscillate about the nominal $(1+0 j)$ point as shown in Fig. 1.

The length of trajectory for a $0.1 \mathrm{rad}$ disturbance is almost identical to the length for a $0.1 \mathrm{pu}$ amplitude disturbance since $\sin (\theta) \approx \theta$ when $\theta$ is small and the phase modulation arc can be approximated to a straight line. A small phase modulation can therefore be analysed in the same way as an amplitude modulation.

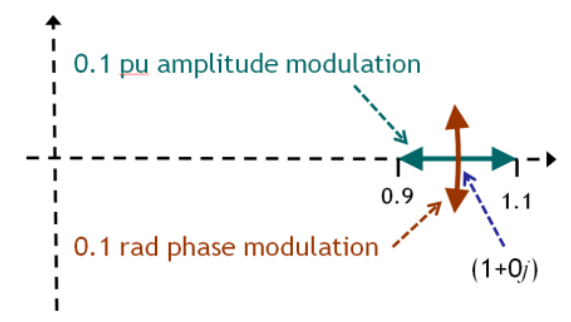

Fig. 1 Phasor oscillation during the bandwidth test

So, the actual vector applied and expected in the presence of either amplitude or phase modulation can be written as:

$$
V=(1+0 j)+M \cos \left(2 \pi f_{M} t\right)
$$

where for amplitude modulation $M=0.1$ is the modulation depth, and for phase modulation $M=0.1 j$. The modulation frequency is $f_{M}$, which can be a maximum of $F_{r}$. Amplitude and phase modulation are applied separately under [2], and can be considered independently and orthogonally [20]. Either amplitude or phase modulation can be considered when determining the filter requirements for TVE. Only phase modulation needs to be considered when determining the requirements for $\mathrm{FE}$ or $\mathrm{RFE}$, since the amplitude modulation has a much smaller impact on frequency or ROCOF perception, explained later using (14).

When the waveform is measured, the deviations from $(1+0 \mathrm{j})$ will be attenuated by the filter (which is assumed to have unity gain at $0 \mathrm{~Hz}$ ). The measured vector will appear as:

$$
V_{\text {Meas }}=(1+0 j)+F\left(f_{M}\right) M \cos \left(2 \pi f_{M} t\right)
$$

where $F\left(f_{M}\right)$ represents the baseband FIR filter response. $F\left(f_{M}\right)$ will have "zero phase" if the filter is symmetric about the timestamp. Also for any filter having only real weights:

$$
F\left(-f_{M}\right)=F *\left(f_{M}\right)
$$

The difference between (2) and (3) reveals the expected voltage phasor measurement error:

$$
V_{\text {Error }}=M\left(F\left(f_{M}\right)-1\right) \cos \left(2 \pi f_{M} t\right)
$$

The TVE error during a 1pu signal application is given by:

$$
T V E=\left|V_{\text {Error }}\right|
$$

and from (5) it is clear to see that TVE will oscillate to maximum values of:

$$
\begin{aligned}
& T V E_{\text {Max }}=\left|M\left(F\left(f_{M}\right)-1\right)\right| \\
& T V E_{\text {Max }}=|M| \times\left|F\left(f_{M}\right)-1\right|
\end{aligned}
$$

Therefore, the constraints on the gain $F\left(f_{M}\right)$ can be derived,

$\left|F\left(f_{M}\right)-1\right|<\frac{T V E_{\text {Limit }}}{|M|}$

Finally, assuming that the filter is symmetric about the timestamp $(t=0)$ in the time domain, the phase of the response of $F\left(f_{M}\right)$ is zero for all $f_{M}$ and so $F\left(f_{M}\right)$ can be regarded as real, not complex. Also, normally $F\left(f_{M}\right)$ will be less than unity at the passband edge. In these cases the maximum TVE at any instant can be expressed as:

$$
1-F\left(f_{M}\right)<\frac{T V E_{\text {Limit }}}{|M|}
$$

Re-ordering and substituting for $|M|=0.1, T V E_{\text {Limit }}=0.03$ $(3 \%)$ reveals:-

$$
\begin{aligned}
& F\left(f_{M}\right)>\left(1-\frac{T V E_{\text {Limit }}}{|M|}\right) \\
& F\left(f_{M}\right)>\left(1-\frac{0.03}{0.1}\right) \text {,i.e. } F\left(f_{M}\right)>0.7
\end{aligned}
$$

This means that the gain of the filter at the edge of the PMU bandwidth $\left(f_{M}=F_{r}\right)$ must be no lower than $-3.098 \mathrm{~dB}$. This $3 \mathrm{~dB}$ figure is not explicitly stated in the C37.118.1 text, but a recent NIST presentation alludes to this figure [19], as does [20], and the $3 \mathrm{~dB}$ point is a conventional way to describe bandwidth. Knowledge of this relationship is very useful for PMU design. For a given filter window length, a relaxed flatness requirement can allow an enhanced attenuation depth outside the passband, and this has clear benefits for broadband harmonic, inter-harmonic and noise rejection.

An asymmetric filter, such as a Kalman filter as proposed in [13] [14], will have a non-zero phase $F\left(f_{M}\right)$ and so the gain at the band edge may need to be higher than $-3 \mathrm{~dB}$ for such a filter, to satisfy (9)-(12).

The analysis can be extended to find filter masks for $F_{F}(f)$ and $F_{R O C O F}(f)$ which need to be complied with to achieve the required FE and RFE limits during the bandwidth test when phase modulation is applied $(M=0.1 j)$ to a 1pu signal magnitude. These limits are given in [2] as shown in TABLE I. 
TABLE I. Bandwidth error limits - C37.118.1a [2]

\begin{tabular}{|c|c|c|c|c|c|c|}
\hline \multirow{2}{*}{\begin{tabular}{|c|}
$\begin{array}{c}\text { F \& ROCOF } \\
\text { performance } \\
\text { limits }\end{array}$ \\
\end{tabular}} & \multicolumn{6}{|c|}{ Error requirements for Compliance } \\
\hline & \multicolumn{3}{|c|}{ P Class } & \multicolumn{3}{|c|}{ MClass } \\
\hline \begin{tabular}{|c|} 
Reporting \\
Rate $\mathrm{F}_{\mathrm{S}}(\mathrm{Hz})$ \\
\end{tabular} & $\mathrm{F}_{f}(\mathrm{~Hz})$ & Max FE & Max RFE & $\mathrm{F}_{\mathrm{f}}(\mathrm{Hz})$ & Max FE & Max RFE \\
\hline 10 & 1 & 0.03 & 0.6 & 2 & 0.12 & 2.3 \\
\hline 12 & 1.2 & 0.04 & 0.8 & 2.4 & 0.14 & 3.3 \\
\hline 15 & 1.5 & 0.05 & 1.3 & 3 & 0.18 & 5.1 \\
\hline 20 & 2 & 0.06 & 2.3 & 4 & 0.24 & 9.0 \\
\hline 25 & 2 & 0.06 & 2.3 & 5 & 0.30 & 14 \\
\hline 30 & 2 & 0.06 & 2.3 & 5 & 0.30 & 14 \\
\hline 50 & 2 & 0.06 & 2.3 & 5 & 0.30 & 14 \\
\hline 60 & 2 & 0.06 & 2.3 & 5 & 0.30 & 14 \\
\hline Formulas & $\min \left(\mathrm{F}_{\mathrm{S}} / 10,2\right)$ & $0.03 * \mathrm{~F}_{\mathrm{f}}$ & $0.18 * \pi^{*} \mathrm{~F}_{\mathrm{f}} 2$ & $\min \left(\mathrm{F}_{s} / 5,5\right)$ & $0.06 * \mathrm{~F}_{\mathrm{r}}$ & $0.18 * \pi * \mathrm{~F}_{\mathrm{r}}^{2}$ \\
\hline
\end{tabular}

For frequency error the analysis extends from (2) and (3) by considering the effect of (specifically) the phase modulation test with with $M=0.1 j$. First the phase error of the measurement can be written as:

$\phi_{\text {Error }}=\angle V_{\text {Meas }}-\angle V$

Assuming $\arctan (\theta) \approx \theta$ for the small values of $|M|$ concerned:

$\phi_{\text {Error }}=\frac{\operatorname{Im}\left[V_{\text {Meas }}\right]}{\operatorname{Re}\left[V_{\text {Meas }}\right]}-\frac{\operatorname{Im}[V]}{\operatorname{Re}[V]}$

Now, using the expressions for $V$ and $V_{\text {Meas }}$ in (2) and (3), it is can be seen that if $M=0.1$, i.e. the case of amplitude modulation, (14) evaluates as (in theory) zero at all times, and so also will the following differentials of phase, to frequency and ROCOF. In reality there may be some small phase errors, and subsequently small FE and RFE during the amplitude modulation test. However, they will be relatively small. By contrast, when phase modulation is applied with $M=0.1 j$, (14) evaluates with a significantly larger and quantifiable response:

$$
\begin{aligned}
& \phi_{\text {Error }}=\frac{F\left(f_{M}\right)|M| \cos \left(2 \pi f_{M} t\right)}{1}-\frac{|M| \cos \left(2 \pi f_{M} t\right)}{1} \\
& \phi_{\text {Error }}=|M|\left(F\left(f_{M}\right)-1\right) \cos \left(2 \pi f_{M} t\right)
\end{aligned}
$$

This can be differentiated to:

$$
\frac{d \phi_{\text {Error }}}{d t}=-2 \pi f_{M}|M|\left(F_{F}\left(f_{M}\right)-1\right) \sin \left(2 \pi f_{M} t\right)
$$

The signed FE will then be:

$$
F E=-f_{M}|M|\left(F_{F}\left(f_{M}\right)-1\right) \sin \left(2 \pi f_{M} t\right)
$$

and this has a maximum absolute (unsigned) value:

$$
|F E|_{\max }=-f_{M}|| M\left|\left(F_{F}\left(f_{M}\right)-1\right)\right|
$$

and by a similar argument to (7)-(11) the lowest gain allowable for a filter $F_{F}(f)$ used within the frequency measurement path, to meet the unsigned FE limit (for a symmetric filter) can be calculated as:
$F_{F}\left(f_{M}\right)>\left(1-\frac{F E_{\text {Limit }}}{f_{M}|M|}\right)$

RFE can be expressed by extending (18):

$R F E=\frac{d F E}{d t}=-2 \pi \cdot f_{M}^{2}|M|\left(F_{R O C O F}\left(f_{M}\right)-1\right) \cos \left(2 \pi f_{M} t\right)$

and again by a similar process this can be used to calculate a limit on the flatness of a filter $F_{\text {ROCOF }}(f)$ used in the ROCOF measurement path

$R F E_{\max }=2 \pi \cdot f_{M}^{2}\left|M\left(F_{R O C O F}\left(f_{M}\right)-1\right)\right|$

$F_{\text {ROCOF }}\left(f_{M}\right)>\left(1-\frac{R F E_{\text {Limit }}}{2 \pi \cdot f_{M}^{2}|M|}\right)$

Some care is required with (20) and (23), since these are exact only if there is no "timestamp shear", i.e. the timestamps (effective window centres after internal calibrations are applied) of the frequency and ROCOF measurement paths are coincident with the timestamp of the main phasor result. If any extra uncompensated filtering is added to the frequency or ROCOF paths, then this eats into the margin available for meeting FE or RFE limits. To illustrate this point, first calculate the instantaneous value of " $\mathrm{dROCOF} / \mathrm{dt}$ " using an extension of the analysis in (2)-(22):

$$
\left(\frac{d R O C O F}{d t}\right)_{\max }=(2 \pi)^{2} \cdot f_{M}^{3}|M|
$$

For example, a $5 \mathrm{~Hz}, 0.1 \mathrm{rad}$ phase modulation in the bandwidth test results in $493 \mathrm{~Hz} / \mathrm{s}^{2}$ ! If the "timestamp shear" of the ROCOF measurement path is even $10 \mathrm{~ms}$, for example due to a single $20 \mathrm{~ms}$ additional boxcar filter that is not perfectly compensated, this results in a $4.9 \mathrm{~Hz} / \mathrm{s}$ error before filter flatness has been considered. Effectively this would reduce $R F E_{\text {Limit }}$ in (23) from the value given in the standard to a lower number, and this would tighten the filter flatness requirement.

Equations (12), (20), and (23) offer three different derivations for the required filter flatness to pass the bandwidth test. There are two approaches. Either, the highest gain (closest to unity) result given by any of the three equations must be taken, and a single filter designed to meet that flatness. Alternatively, separate filters can be designed and implemented within a single PMU to produce the phasor, frequency, and ROCOF results. The masks constraining $F_{F}(f)$ for frequency (20), and $F_{\text {ROCOF }}(f)$ for ROCOF (23) generally allow $>3 \mathrm{~dB}$ of attenuation at the band edge. Knowledge of this option is extremely useful, and can be exploited to tailor the performance of PMUs. Examples are shown later.

Since the passband flatness gain is referred to the unity gain at $0 \mathrm{~Hz}$, and the PMU is assumed to be a linear device, then the bandwidth test could in theory be carried out with any fundamental phasor magnitude and phase, as an alternative to $(1+0 \mathrm{j})$, so long as the modulation amplitude is kept at $10 \%$ of the fundamental amplitude, and the phase modulation is kept at $0.1 \mathrm{rad}$. There would be no effect on the filter mask derivation. However, the C37.118.1a test procedure [21] carries out the

This is a postprint of a paper submitted to and accepted for publication in IEEE Transactions on Instrumentation and Measurement [http://dx.doi.org/10.1109/TIM.2015.2445111] and is subject to IEEE copyright. 
tests at nominal (1pu) magnitude because it minimises the risk of non-linear saturation effects, and also means the PMU is operating at almost its optimum SNR (Signal to Noise ratio). A similar argument applies later to the OOB test, where the analysis would be unaltered for a fundamental of different amplitude or phase, so long as the interfering interharmonic was still at $10 \%$ of the fundamental magnitude.

\section{A. Bandwidth in the Reference algorithm}

The Reference algorithm in C37.118.1a uses a flatness mask in the passband of $\pm 0.043 \mathrm{~dB}$ as part of its filter design process (Fig. 2). This value coincides with an error of $0.5 \%$ in absolute amplitude calibration. By using such a flat filter, as the fundamental frequency varies over the passband, no extra amplitude calibration needs to be calculated for the digital filter response, in order to meet a TVE of $1 \%$. This makes real-time computation easier within the Reference algorithm. Nevertheless, as was shown above, a flatness of $\pm 0.043 \mathrm{~dB}$ is far tighter than is required to pass the bandwidth test, in terms of TVE, Frequency and ROCOF requirements.

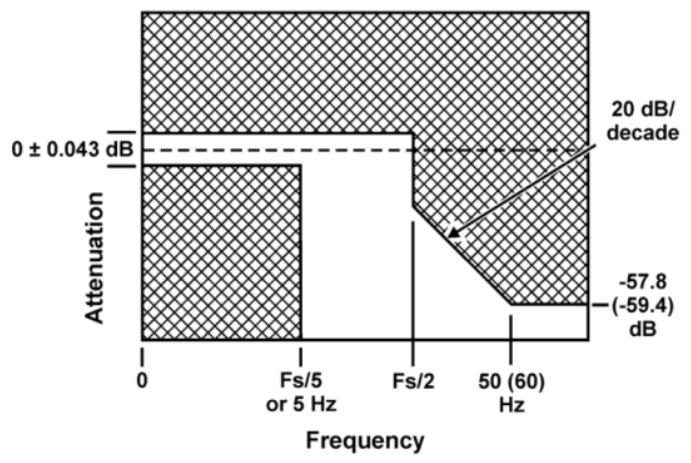

Fig. 2 Filter mask for the C37.118.1a Reference algorithm [2]

\section{B. Bandwidth in a frequency-tracking or frequency- compensated algorithm.}

If the filter amplitude response is known and can be expressed mathematically using a fitted function or a lookup table, then the off-nominal filter gain can be accounted for, using the measured frequency value. This could be called a frequency-compensated PMU.

Alternatively, and additionally, if the quadrature oscillator [1] [4] tracks the measured fundamental frequency, so that the fundamental input frequency to the FIR filters is always moved towards $0 \mathrm{~Hz}$, then the deviation of the filter gain from unity is minimised, even during off-nominal frequency. This is a frequency-tracking PMU [4] [5] . Combining both approaches can lead to very low values of steady-state TVE as the fundamental frequency changes, but allows a relaxation of the flatness from $\pm 0.043 \mathrm{~dB}$ to nearly the $3 \mathrm{~dB}$ figure derived above.

In practice, it is found that setting a target phasor filter gain at the passband edge of about $-2.5 \mathrm{~dB}$ leads to acceptable results for phasors in the bandwidth test, with highest TVE results in the region of $2.3-2.75 \%$. This margin gives a very low risk of unexpected compliance failures due to ADC resolution or noise since these tests are done with $1 \mathrm{pu}$ signals and all the modulated signal is in the passband. Filter gains for the frequency and ROCOF paths can be reduced below $-2.5 \mathrm{~dB}$ by (20), and (23).

\section{OUT OF BAND (OOB); STOPBAND REJECTION}

The other major change in C37.118.1a is within the OOB test, and requires great care in the filter design. In the original C37.118.1, the emphasis was on achieving a 1.3\% TVE result when the $10 \%$ OOB interharmonics were applied. This corresponded to a $20 \mathrm{~dB}$ stopband rejection, which defined the original Reference algorithm mask. However, C37.118.1 also contained OOB FE and RFE limits that could not be met by its Reference algorithm, with just $20 \mathrm{~dB}$ stopband rejection.

In C37.118.1a, the OOB RFE limit has been suspended. But, the OOB FE limit of $0.01 \mathrm{~Hz}$ has been retained. This is significant because it requires $>>20 \mathrm{~dB}$ of stopband rejection In fact, it requires stopband rejection in excess of $40 \mathrm{~dB}$. Therefore, it is now the OOB FE limit, not the 1.3\% TVE limit, which defines the filter mask given in C37.118.1a. If different filter designs are used for the phasor, frequency and ROCOF calculations, then it is the filter used for the frequency output that is most relevant during this test. If the filter used in the phasor measurement path also has $>40 \mathrm{~dB}$ rejection, then the TVE errors during the OOB test will be much less than the $1.3 \%$ limit and should pass "by default" if the steady-state PMU performance tests also pass. The new mask for the Reference algorithm is shown in Fig. 2. To achieve the increased stopband rejection, using the Reference algorithm, requires longer time windows and slightly adjusted filters compared to those given in C37.118.1. This has, in turn, required longer (relaxed) response and latency limits to be applied in C37.118.1a. Some PMUs may exhibit significantly shorter responses and latencies than the C37.118.1a limits.

\section{A. Determining the required $O O B$ filter rejection}

It is possible to calculate the required baseband filter rejection $F()$, which is a function of frequency separation from the tuned frequency $f_{T}$. (For a fixed-filter PMU $f_{T}=f_{0}$, and for a tracking $\operatorname{PMU} f_{T} \approx f$ ).

If a positive-sequence interharmonic of amplitude $A$ is applied at frequency $f_{I H}$, while the fundamental signal has positive-sequence amplitude $1 \mathrm{pu}$ at frequency $f$ :

$$
V_{\text {in }}=e^{j 2 \pi f t}+A e^{j 2 \pi f_{I H} t}
$$

When the heterodyning with the quadrature oscillator and then filtering $F()$ are applied (see Fig. 1 in [4]), with both tuned to expect a fundamental frequency $f_{T}$, the perceived measurement vector at the filter output will consist of the dominant fundamental with a magnitude $\approx 1 \mathrm{pu}$, rotating at a rate of $2 \pi\left(f-f_{T}\right) t \mathrm{rad} / \mathrm{s}$, upon which is superimposed another circular trajectory of amplitude $A$, with a rotation rate of $2 \pi\left(f_{I H^{-}} f_{T}\right) t \mathrm{rad} / \mathrm{s}$ :

$$
\begin{aligned}
& V_{\text {Heterodyned }}=e^{-j 2 \pi f_{T} t}\left(e^{j 2 \pi f t}+A e^{j 2 \pi f_{I H} t}\right) \\
& V_{\text {Heterodyned }}=e^{j 2 \pi\left(f-f_{T}\right) t}+A e^{j 2 \pi\left(f_{I H}-f_{T}\right) t}
\end{aligned}
$$

This is a postprint of a paper submitted to and accepted for publication in IEEE Transactions on Instrumentation and Measurement [http://dx.doi.org/10.1109/TIM.2015.2445111] and is subject to IEEE copyright. 


$$
\begin{aligned}
V_{\text {Meas }} & =F\left(f-f_{T}\right) e^{j 2 \pi\left(f-f_{T}\right) t} \\
& +F\left(f_{I H}-f_{T}\right) A e^{j 2 \pi\left(f_{I H}-f_{T}\right) t} \\
V_{\text {Meas }} & =e^{j 2 \pi\left(f-f_{T}\right) t} \times \\
& {\left[F\left(f-f_{T}\right)+A F\left(f_{I H}-f_{T}\right) e^{j 2 \pi\left(f_{I H}-f\right) t}\right] }
\end{aligned}
$$

During the OOB test the fundamental is only varied over a small range from $f_{0}$, so the fundamental should be well within the passband. Therefore, it is safe to assume that the filter gain $F\left(f-f_{T}\right)$ is close enough to unity to ignore (in a frequencytracking PMU $f=f_{T}$ an so $F\left(f-f_{T}\right)=1$ exactly). Therefore, the measurement error is due to the second term. Frequency error cannot be evaluated by directly examining $V_{\text {Meas }}$ since this vector is expected to be rotating around the origin with an amplitude of roughly $1 \mathrm{pu}$ at a rate of $2 \pi\left(f-f_{T}\right) t \mathrm{rad} / \mathrm{s}$. So, in order to assess frequency error, a new vector $\Delta V_{\text {Meas }}$ can be defined which maintains a steady-state value when only the fundamental is present. $\Delta V_{\text {Meas }}$ is defined simply by heterodyning $V_{\text {Meas }}$ by $-2 \pi\left(f-f_{T}\right) t$ to bring it nominally onto the point $(1+0 \mathrm{j})$, plus the disturbance due to the OOB interharmonic.

$$
\begin{aligned}
\Delta V_{\text {Meas }} & =V_{\text {Meas }} e^{-j 2 \pi\left(f-f_{T}\right) t} \\
\Delta V_{\text {Meas }} & =\left(1+A F\left(f_{I H}-f_{T}\right) e^{j 2 \pi\left(f_{I H}-f\right) t}\right)
\end{aligned}
$$

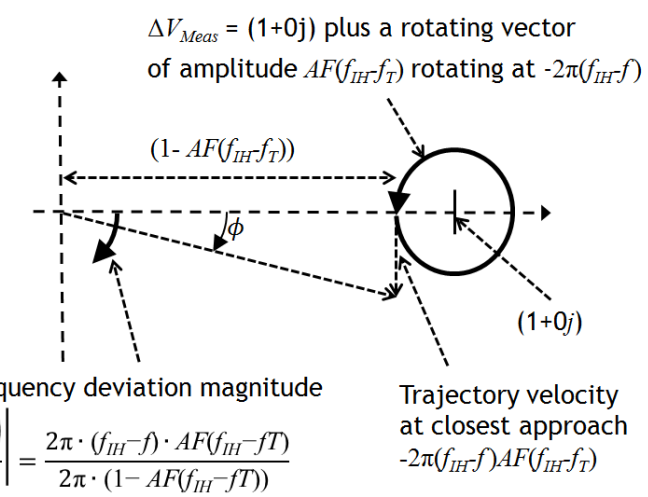

Fig. 3. $\Delta V_{\text {Meas }}$ and the Peak frequency error occurring during the Out-OfBand tests.

It can be seen (Fig. 3) that $\Delta V_{\text {Meas }}$ consists of a small circular error trajectory centred on the point $(1+0 \mathrm{j})$. The effect on frequency measurement is determined by the maximum rate of change of phase error $\Delta \phi$ :

$$
\Delta \phi=\angle\left(\Delta V_{\text {Meas }}\right)
$$

The biggest rate of change of $\Delta \phi$ will occur at the closest approach of $\Delta V_{\text {Meas }}$ to the origin $(0+0 j)$, when $2 \pi\left(f_{I H} f\right) t=\pi$. At this instant $\Delta V_{\text {Meas }}$ has its lowest magnitude, with a value of $\left(\left(1-A F\left(f_{I H}-f_{T}\right)\right)+0 \mathrm{j}\right)$, but also has its maximum velocity parallel to the $j$ axis: $-2 \pi\left(f_{I H^{-}} f\right) A F\left(f_{I H^{-}} f_{T}\right) \mathrm{s}^{-1}$. Therefore, the maximum magnitude of the rate of change of phase error $\Delta \phi$ is:

$$
\begin{aligned}
& \left|\frac{d(\Delta \phi)}{d t}\right|_{\text {Max }}=\left|\frac{-2 \pi\left(f_{I H}-f\right) \cdot A \cdot F\left(f_{I H}-f_{T}\right)}{\left(1-A \cdot F\left(f_{I H}-f_{T}\right)\right)}\right| \\
& F E_{\text {Max }}=\frac{1}{2 \pi} \cdot\left|\frac{d(\Delta \phi)}{d t}\right|_{\text {Max }}
\end{aligned}
$$

and therefore:

$$
F\left(f_{I H}-f_{T}\right)<\frac{F E_{\text {Max }}}{A\left(\left|f_{I H}-f\right|+F E_{\text {Max }}\right)}
$$

Since $F E_{\text {Max }}=0.01 \mathrm{~Hz}$, and the closest OOB frequency for the $F_{S}=10 \mathrm{~Hz}$ PMU is $5 \mathrm{~Hz}, F E_{M a x}<<\left|f_{I H} f\right|$ and so the following approximation is valid:

$$
F\left(f_{I H}-f_{T}\right)<\frac{F E_{M a x}}{A \cdot\left|f_{I H}-f\right|}
$$

This defines the highest filter gain that can possibly satisfy the OOB test. In practice, to allow a safety margin for noise, Effective Number of Bits (ENOB) of ADC resolution etc., it is sensible to allow reduce this figure by $3 \mathrm{~dB}$, otherwise, any imperfection or noise within signal conditioning or sampling hardware may cause a failure in the OOB test. Also, to allow for the fact that the PMU frequency measurement may be made with a different filter window than the phasor measurement is made, and noting that in the OOB test the frequency error limit is the defining constraint, we recognise that the key filter to be defined is actually $F_{F}(f)$ and not $F(f)$

$F_{F}\left(f_{I H}-f_{T}\right)<\left(\frac{F E_{\text {Max }}}{A \cdot\left|f_{I H}-f\right|}\right) 10^{\left(\frac{-3}{20}\right)}$

However, the way that (37) needs to be evaluated is different for fixed-filter and frequency-tracking PMUs, as is the range of values of $f_{I H}$ which should in theory be applied to validate the performance.

\section{B. Fixed-filter algorithms}

For any interharmonic such as flicker at frequency $f_{I H}$, the ripple frequency of error at the PMU output, due to the heterodyning process, is given by:

$$
f_{\text {ripple }}=\left|f_{I H}-f_{T}\right|
$$

The aim of the C37.118.1a OOB test is to verify a filter stopband which limits any measurement ripples with frequencies $>=F_{S} / 2$. Therefore:

$\left|f_{I H}-f_{T}\right| \geq \frac{F_{S}}{2}$

For fixed-filter PMUs such as the Reference algorithm, $f_{T}=f_{0}$ always, and so:

Fixed-filter stopband : $\left|f_{I H}-f_{0}\right| \geq \frac{F_{S}}{2}$

This matches exactly with the C37.118.1a OOB test, where the stopband is defined exactly as (40). C37.118.1a testing applies (40) but only varies fundamental frequency over a reduced range compared to the whole valid range $\left(f_{0} \pm F_{r}\right)$ :

This is a postprint of a paper submitted to and accepted for publication in IEEE Transactions on Instrumentation and Measurement [http://dx.doi.org/10.1109/TIM.2015.2445111] and is subject to IEEE copyright. 


$$
f \text { range: }\left(f_{0}-\frac{F_{S}}{20}\right) \leq f \leq\left(f_{0}+\frac{F_{S}}{20}\right)
$$

For fixed-filter devices, on the left hand side (LHS) of (37), the mask always refers to $F_{F}\left(f_{I H}-f_{0}\right)$. But, on the right hand side (RHS), there is a worst case during C37.118.1 a testing where $\left(f_{I H}-f\right)$ reaches $\left(F_{S} / 2+F_{S} / 20\right)=11 F_{S} / 20$, i.e. a $10 \%$ increase from $F_{S} / 2$. This $10 \%$ increase means that the gain $F_{F}()$ needs to be $20 * \log (1.1)=0.83 \mathrm{~dB}$ reduced from a value obtained via a more "naïve" (and incorrect) analysis where $f_{0}$ is substituted for both $f$ and $f_{\mathrm{T}}$ in (37)):

$$
F_{F}\left(f_{I H}-f_{0}\right)<\left(\frac{F E_{\text {Max }}}{A \cdot\left|f_{I H}-f_{0}\right|}\right) 10^{\left(\frac{-3}{20}\right)}
$$

Careful evaluation of (37), with $f_{T}=f_{0}$ and over the range (41), leads to the mask given for the C37.118.1a Reference algorithm.

Note that if the full valid fundamental input frequency range $\left(f_{0} \pm F_{r}\right)$ was tested or explored, the $0.83 \mathrm{~dB}$ factor could rise to $20 * \log (1.4)=2.92 \mathrm{~dB}$ with $F_{r}=F_{S} / 5$ to achieve the same FE using the fixed-filter device.

\section{Frequency-tracking algorithms}

The effect and analysis of interharmonics within a frequency-tracking device is different. In such a device, $f_{T}=f$ if the algorithm is tracking correctly. Therefore, to define the required stopband from (38) and (39):

$$
\text { Tracking stopband : }\left|f_{I H}-f\right| \geq \frac{F_{S}}{2}
$$

Equation (43) implies that a different regime from (40) should ideally be used to test a frequency-tracking device. The difference between $(40)$ and (43) is small when $f \approx f_{0}$ but becomes significant as the fundamental frequency $f$ deviates from nominal.

The actual C37.118.1a test applies the regime in (40) and (41). The issue here is that the lowest ripple frequency appearing after the heterodyning during the test regime is given by (38) with (as a worst-case example) $f_{T}=f=\left(f_{0}+F_{S} / 20\right) \mathrm{Hz}$ and $f_{I H}=\left(f_{0}+F_{S} / 2\right) \mathrm{Hz}$. This requires a stopband start frequency of only $9 F_{S} / 20 \mathrm{~Hz}$, which is $10 \%$ less than the stopband start frequency required using a fixed-filter device to pass the same test.

Therefore, it is harder for a frequency-tracking algorithm to pass the C37.118.1a OOB test than for a fixed-filter algorithm. The filter has to be more aggressively designed to achieve stopband cutoff at a baseband filter frequency that is $10 \%$ lower than the equivalent fixed-filter design. Making use of the available $\sim 2.5 \mathrm{~dB}$ filter flatness relaxation is one way to try to achieve this more aggressive cutoff.

Notice that if the testing regime was changed to (43) for the frequency-tracking device, this $10 \%$ narrowing would no longer be required. Also, in that case, the full valid fundamental input frequency range $\left(f_{0} \pm F_{r}\right)$ (and potentially much wider) could be tested, using the same baseband filter design, and the same $0.01 \mathrm{~Hz} F E$ might still be attained, or nearly attained (but see section $\mathrm{D}$ below).

For example, in the following valid UK network scenario, a frequency-tracking PMU with $f_{0}=50 \mathrm{~Hz}$ and $F_{S}=10 \mathrm{~Hz}$ would be able to successfully measure a fundamental signal at $f=47 \mathrm{~Hz}$, because it's filter would be centred on $47 \mathrm{~Hz}$, with a bandwidth of $\pm 2 \mathrm{~Hz}(45-49 \mathrm{~Hz})$, and a stopband of $<=42 \mathrm{~Hz}$ and $>=52 \mathrm{~Hz}$. A fixed-filter equivalent PMU would not be able to make such a measurement so well, since it's filter would be centred on $50 \mathrm{~Hz}$ and the $47 \mathrm{~Hz}$ fundamental would be outside the $\pm 2 \mathrm{~Hz}, 3 \mathrm{~dB}$ bandwidth and almost in the stopband. However a $52 \mathrm{~Hz}$ flicker interharmonic would be touching the passband of the fixed-filter device. The filtering would be quite asymmetric about the fundamental.

The required filter attenuation can again be evaluated by (37). For a frequency-tracking PMU, $f_{T}=f$ if the algorithm is tracking correctly. This means there is no deviation between the frequencies on the LHS and RHS of (37) and the $0.83 \mathrm{~dB}$ factor is not required. In fact, the gain of the filter can be $0.92 \mathrm{~dB}$ higher at the lowest required frequency offset from the filter tuned frequency $\left(f_{I H}-f_{T}\right)$, than the simplistic analysis of (42) suggests, since that minimum frequency offset (the stopband start frequency) $\left(f_{I H}-f\right)$ is reduced by $10 \%$ compared to $\left(f_{I H}-f_{0}\right)$. to so that it is only 0.9 times $\left(f_{I H}-f_{0}\right)$. The required attenuation is therefore $20 * \log 10(1 / 0.9)=0.92 \mathrm{~dB}$ higher than equation (42) would predict. This works slightly in favour of the tracking PMU during the OOB test, but not enough to balance the difficulty introduced by the $10 \%$ stopband reduction.

\section{Accounting for adaptive filter shortening}

There is a further complication for tracking algorithms, which is that the entire filter window design may adapt linearly to become shorter as the measured fundamental frequency increases (to track the filter notches with the harmonics). Tracking algorithms such as [4] [5] fall into this category. This will expand the filter frequency response proportionately. Therefore, when designing such an adaptive filter for the baseline $f_{0}$ case, the mask window width in frequency may need to be further reduced by a proportional factor of:

$$
\text { Adaptive MaskWidth Factor }=f_{0} /\left(f_{0}+\frac{F_{S}}{20}\right)
$$

Otherwise, a tracking PMU will likely fail an OOB test when $f=\left(f_{0}+F_{S} / 20\right)$. Examples of the factor in (44) are 0.99 (a $1 \%$ reduction) for $F_{S}=10 \mathrm{~Hz}$ and 0.952 (a $4.8 \%$ reduction) for $F_{S}=50 \mathrm{~Hz}$, with $f_{0}=50 \mathrm{~Hz}$. This reduction is not due to the C37.118.1a test process regime, but is inherent in a frequencytracking device with such adaptive filters. If a wider fundamental input frequency range $\left(f_{0} \pm F_{r}\right)$ was tested, the reductions would need to be increased accordingly, irrespective of whether regime (40) or (43) was used, in order to pass the OOB test. For example over the whole valid range with $f_{0}=50 \mathrm{~Hz}$ and $F_{r}=5 \mathrm{~Hz}$, the factor reduces to 0.909 , a $9 \%$ reduction. 


\section{E. Overall effects on the filter mask for frequency-tracking algorithms}

The total effect is that the stopband start frequency for a frequency-tracking algorithm is reduced by between $11 \%$ and $14.8 \%$ compared to the mask for a fixed filter, when the filter is designed at $f_{0}$ and the $\mathrm{C} 37.118 .1 \mathrm{a}$ OOB test procedure is to be applied. As an example, Fig. 4 shows the masks and typical filter responses for the fixed-filter C37.118.1a Reference algorithm, and a C37.118.1a-compliant tracking algorithm [15], in the $f=f_{0}=F_{S}=50 \mathrm{~Hz}$ case. The mask frequencywidth reductions required for the tracking filter $(10 \%+4.8 \%)$ have been indicated, showing how the mask stopband begins almost $15 \%$ closer to the passband than $F_{S} / 2$. Both masks are calculated using (37) and the procedures of section VI B thru VI.D. Fig. 5 shows detail around the critical cutoff point, including the annotations for the $0.83 \mathrm{~dB}$ and $0.92 \mathrm{~dB}$ deviations from the "naïve" analysis resulting from (42).
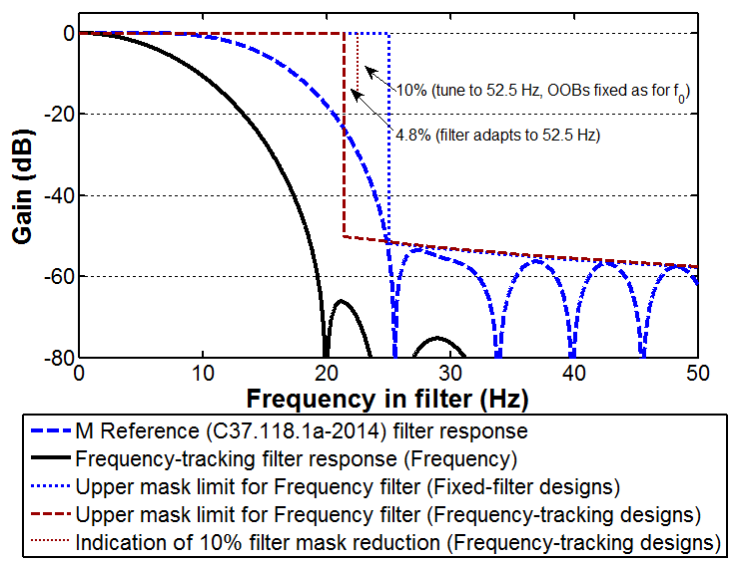

Fig. 4 Example of filter masks and responses for the fixed-filter Reference algorithm, and a C37.118.1a-compliant tracking algorithm. $f=f_{0}=F_{S}=50 \mathrm{~Hz}$

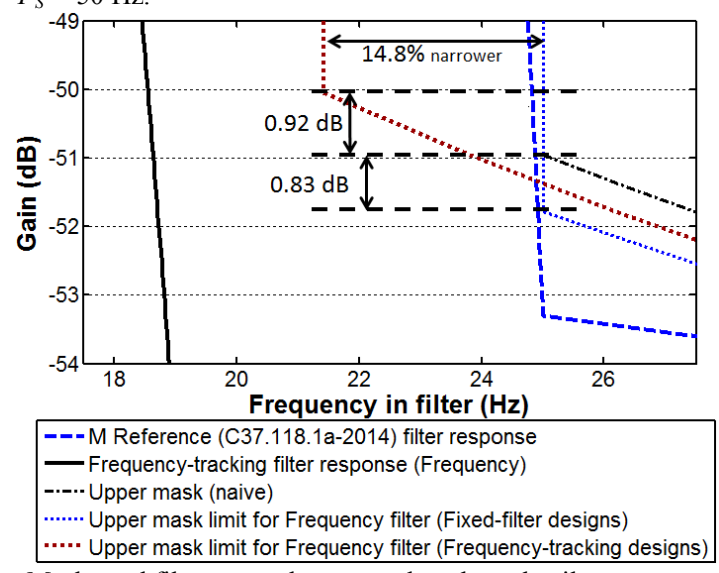

Fig. 5 Masks and filter examples zoomed to show detail.

$f=f_{0}=F_{S}=50 \mathrm{~Hz}$

\section{EXAMPLE APPLICATION OF MASKS FOR THE DESIGN OF THE REPORTING-RATE $25 \mathrm{~Hz}$ PMU}

The benefit of using the design equations (12), (20), (23) and (37) can be demonstrated by showing an example with the reporting rate $25 \mathrm{~Hz}$ PMU. This PMU is the most difficult of all the frequency-tracking PMUs to design. This is because it is the lowest reporting-rate PMU that must achieve the full $5 \mathrm{~Hz}$ bandwidth, while at the same time providing a stopband start frequency at (nominally) $12.5 \mathrm{~Hz}$. This means that, for the frequency-tracking device, the design mask stopband start frequency actually needs to be $10 \%+2.4 \%$ (by (44)), i.e. a full $12.4 \%$ less than $12.5 \mathrm{~Hz}$.

The example shows the design masks for a fixed-filter PMU, and a frequency-tracking PMU. The Reference algorithm from [2] is used as the filter example which fits the fixed-filter mask. The frequency-tracking PMU from [15] is used as the filter examples which have been designed to fit the frequency-tracking masks for C37.118.1a(2014). This PMU algorithm and filter designs follow the principles laid down in the earlier papers [4] and [5]. In particular, [5] presented an Mclass C37.118.1(2011)-compliant device with reporting-rate 50 Hz. However, due to the amendments in C37.118.1a(2014), and to provide compliance with other reporting rates, the number of filter stages and the lengths of filters needed to be increased relative to [5], with customised designs for each reporting rate, and a combination of delicate calibration and filter-shaping techniques. The precise algorithm and filter configuration is beyond the scope of this particular paper. What is important for this paper is to demonstrate that the filter masks can be used to design the devices, and to show that the device is then compliant if it meets the mask. In the frequencytracking example, the option to use different filters for the phasor, frequency and ROCOF paths is exploited. All three paths have different required responses in the passband to fit (12), (20), and (23), which define the lower mask limits within the passband. Meanwhile, the filter used in the frequency path is of primary concern during the OOB test, which defines the mask upper limit in the stopband.

Exactly how a PMU implements 3 different window functions (filters) for the three paths, is of course extremely specific to the detailed PMU internal design, which is not in the direct scope of this paper. In general, however, such a strategy does not require the computational complexity to increase by a factor of 3, with parallel architectures for the 3 paths. There are several methods by which all three filter path shapes (and more, if needed) can be derived from a single "mother" window function without any significant computation overload, compared to a "normal" single-window-function measurement. One simple example is given by equations C.3 and C.4 within the Reference PMU described by [2]. These equations describe post-processing of the primary window/filter results in order to create frequency and ROCOF measurement paths with more robust filtering against noise etc. In the frequency domain, these processes create different responses for the three measurement paths: phasor, frequency and ROCOF, with only a minimal computation overhead. The approach can be generalised by allowing all three paths to apply post-processing to a single "mother" window/analysis, to create customised frequency-domain responses which comply with the masks derived in this paper. Descriptions of the myriad of mathematical options to do this are outside the scope of this paper, which concentrates on the filter shape requirements, not how to achieve the filter shapes.

Firstly, Fig. 6 shows the passband masks and example filter responses. The 3 different masks required to meet TVE, FE and RFE requirements in the bandwidth test, by (12), (20), and (23), are shown. The fixed-filter example uses only a single

This is a postprint of a paper submitted to and accepted for publication in IEEE Transactions on Instrumentation and Measurement [http://dx.doi.org/10.1109/TIM.2015.2445111] and is subject to IEEE copyright. 
filter to meet all three requirements. However, the frequencytracking example has different filters for the 3 paths (phasors, frequency and ROCOF). The allowed attenuation for frequency and ROCOF filter paths is significantly higher than that in the main phasor path which must be $3 \mathrm{~dB}$ or less. To create the flattest responses in the phasor and frequency paths requires the use of negative weights in the filter, as shown in Fig. 7. However, the ROCOF filter flatness can often be relaxed so far that negative weights are no longer required. This has large benefits for wideband noise and (inter)harmonic rejection, as will be shown.

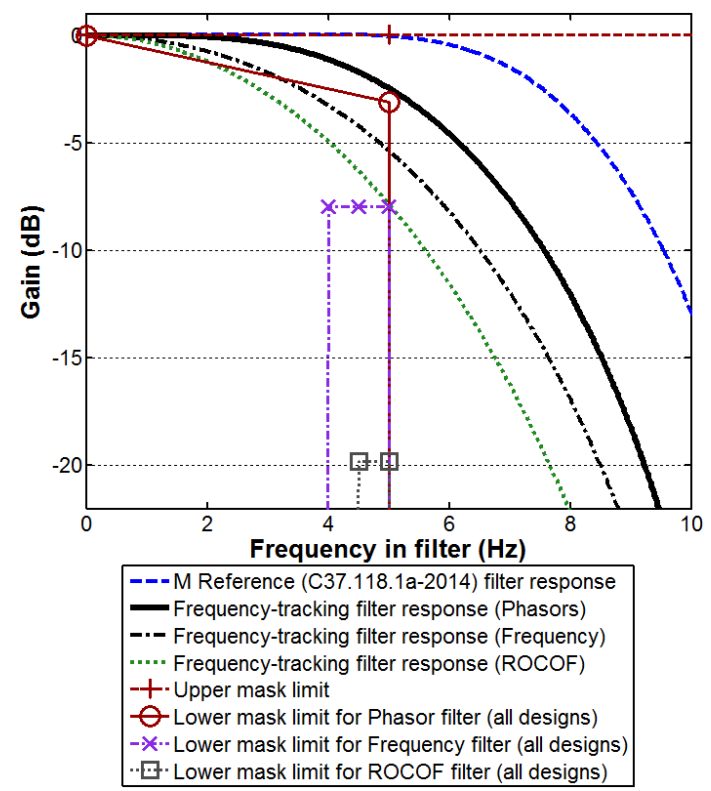

Fig. 6 Masks for the passband to meet the bandwidth test requirements. $f=f_{0}=50 \mathrm{~Hz} . F_{S}=25 \mathrm{~Hz}$.

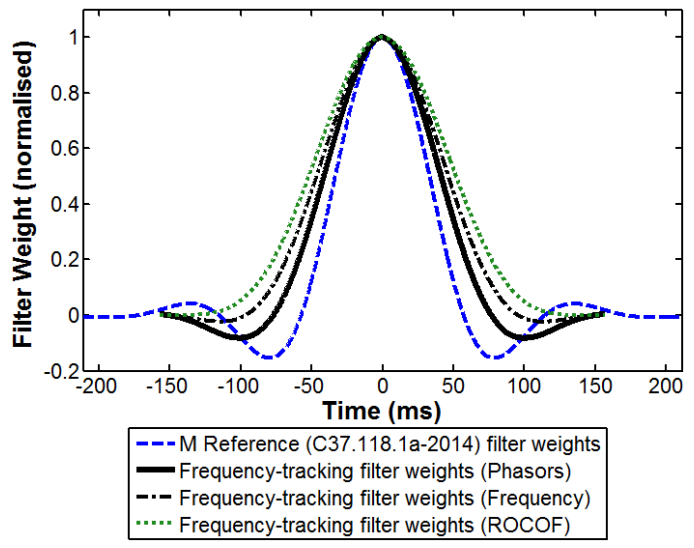

Fig. 7 Filter weight examples for the fixed-filter PMU and the frequencytracking PMU. $f=f_{0}=50 \mathrm{~Hz} . F_{S}=25 \mathrm{~Hz}$.

Even the slight relaxation in the flatness of the frequencytracking PMU frequency-path filter response (Fig. 6) is useful, since it allows a faster rolloff towards the stopband, where the filter in the frequency-measurement path is of paramount importance. Fig. 8 shows the region around the critical corner frequency where the OOB stop-band begins. The fixed-filter Reference design just meets the fixed-filter mask. The phasorpath filter of the frequency-tracking device just meets the frequency-tracking mask, but really it is the frequency-path response that must meet this mask. Fig. 8 shows there to be a $5 \mathrm{~dB}$ margin, which has been "bought" by relaxing the passband flatness of the frequency-path filter. The mask width (in frequency) for the frequency-tracking device with $F_{S}=25 \mathrm{~Hz}$ is $12.4 \%$ reduced compared the fixed-filter device mask, by (43) and (44). The ROCOF-path filter response also appears on Fig. 8, and is yet further below the frequency-path response. Therefore, it can be seen that by relaxing the filter flatness of the frequency-path and ROCOF-path filters in the passband, their stopband attenuation is significantly improved. The effect is even more marked at higher wideband harmonic and interharmonic frequencies, as shown in Fig. 9 and Fig. 10.

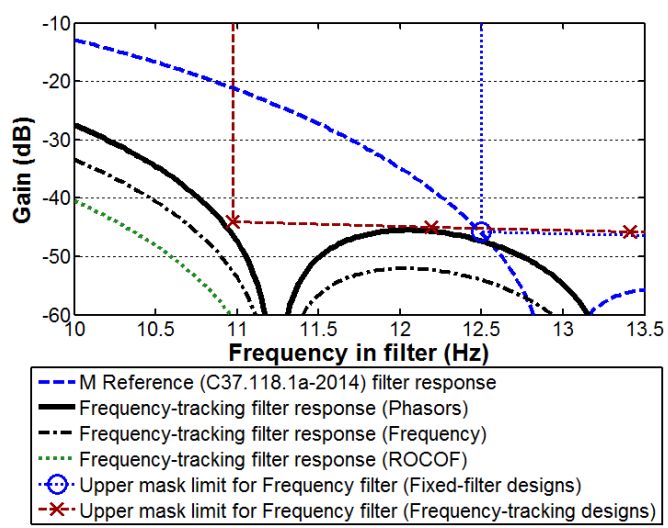

Fig. 8 OOB mask and responses for the fixed-filter PMU and the frequencytracking PMU. $f=f_{0}=50 \mathrm{~Hz} . F_{S}=25 \mathrm{~Hz}$.

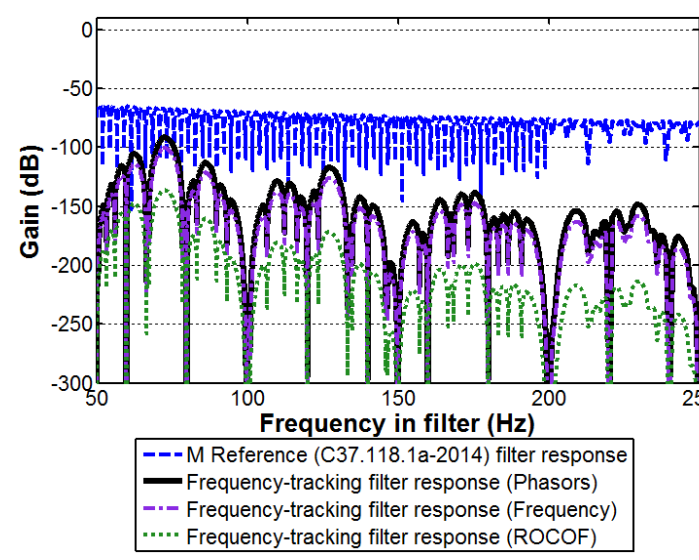

Fig. 9 Filter responses between 50 and $250 \mathrm{~Hz}$ for the fixed-filter PMU and the frequency-tracking PMU. $f=f_{0}=50 \mathrm{~Hz} . F_{S}=25 \mathrm{~Hz}$.

This is a postprint of a paper submitted to and accepted for publication in IEEE Transactions on Instrumentation and Measurement [http://dx.doi.org/10.1109/TIM.2015.2445111] and is subject to IEEE copyright. 


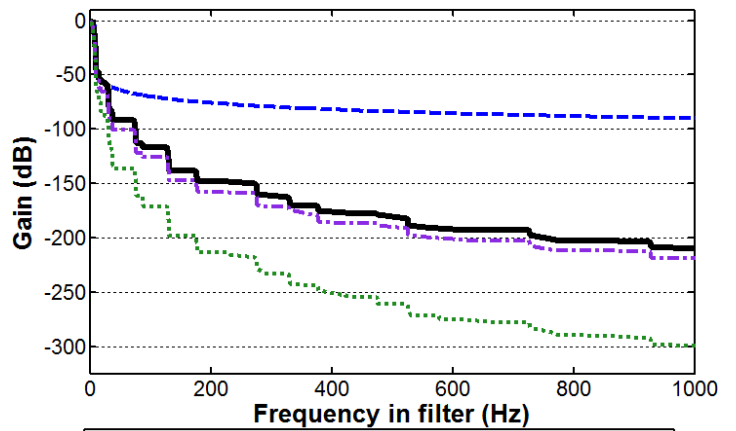

---M Reference (C37.118.1a-2014) filter response envelope - Frequency-tracking filter response envelope (Phasors) - - Frequency-tracking filter response envelope (Frequency) ..... Frequency-tracking filter response envelope (ROCOF)

Fig. 10 Filter response envelopes between 0 and $1 \mathrm{kHz}$ for the fixed-filter PMU and the frequency-tracking PMU. $f=f_{0}=50 \mathrm{~Hz} . F_{S}=25 \mathrm{~Hz}$.

\section{EXAMPLE TEST RESULTS FOR PMUS DESIGNED USING THE MASKS}

This section shows test results (in simulation) from the bandwidth and OOB tests for the Reference (fixed-filter) PMU algorithm and the frequency-tracking PMU algorithm. The fixed-filter mask described in this paper was used by the creators of the Reference PMU, while the frequency-tracking masks were used to help design the frequency-tracking PMU [15]. Therefore, if the PMUs all pass the tests, this is a good indication that the masks accurately represent the requirements. The results of PMUs in the bandwidth test are shown in Fig. 11 (TVE) , Fig. 12 (FE) and Fig. 13 (RFE). The results are the worst-case maximum errors for any report in each test for each PMU, and are all obtained when the maximum modulation frequency $F_{r}$ is applied to each PMU during its specific test. This is to be expected for a most PMUs, since filter gain tends to roll off towards the edge of the passband.

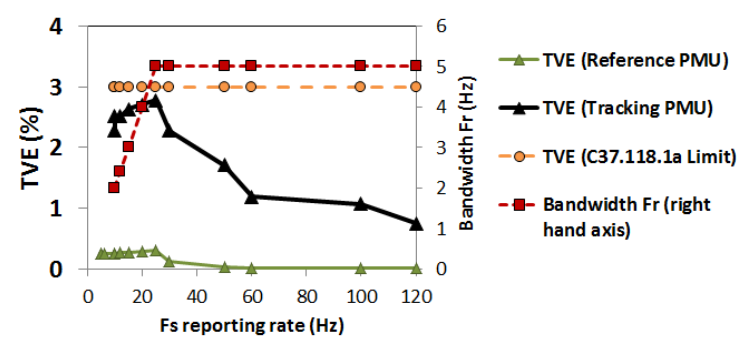

Fig. 11. Maximum TVE results during bandwidth tests

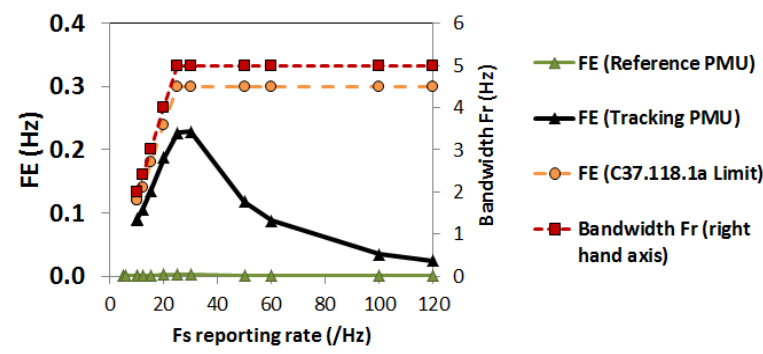

Fig. 12. Maximum Frequency Error (FE) results during bandwidth tests

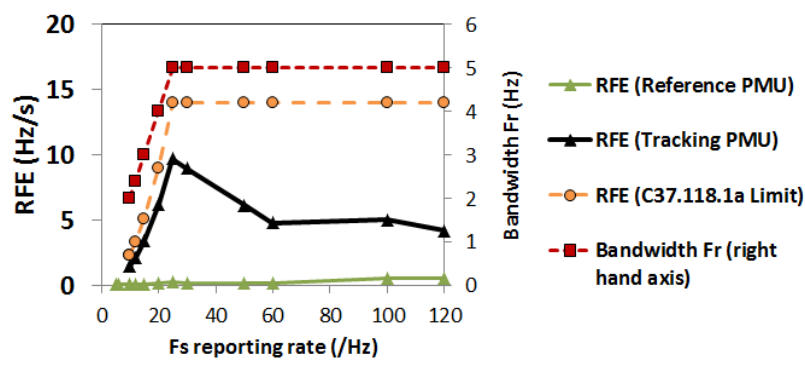

Fig. 13. Maximum Rate of Change of Frequency Error (RFE) results during bandwidth tests

The worst-case TVE, FE and RFE results during the OOB test are similarly presented in Fig. 14, Fig. 15 and Fig. 16. The evidence from testing shows that the filter mask for the frequency-tracking device is appropriate, and can indeed be used to design filters that are compliant when tested within PMU algorithms and subjected to C37.118.1 a testing. Intimate knowledge of these masks removes much of the "trial and error" process of designing a compliant PMU. A full set of C37.118.1a test results (in simulation, for both $f_{0}=50 \mathrm{~Hz}$ and $f_{0}=60 \mathrm{~Hz}$ devices) using the same frequency-tracking design methodology is available at [15].

The test results in Fig. 11-Fig. 16 clearly show that the Reference (fixed-filter) PMU demonstrates a flatter passband than the frequency-tracking device. However, the frequencytracking device has a much better stopband rejection than the fixed-filter device, within the frequency and ROCOF measurement paths. These results are exactly what is expected following the example mask-generation process described in section VII.

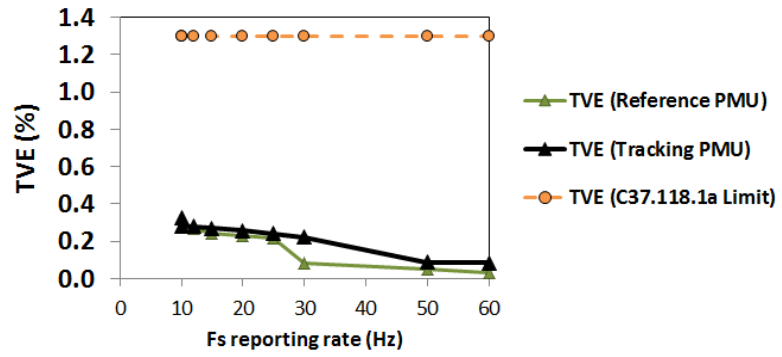

Fig. 14. Maximum TVE results during OOB tests

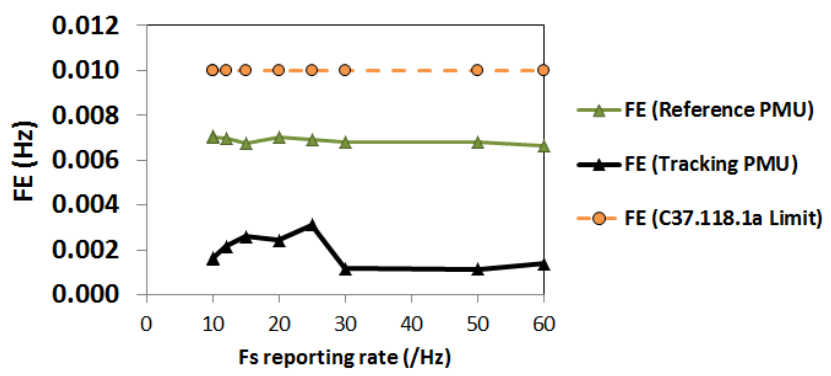

Fig. 15. Maximum Frequency Error (FE) results during OOB tests

This is a postprint of a paper submitted to and accepted for publication in IEEE Transactions on Instrumentation and Measurement [http://dx.doi.org/10.1109/TIM.2015.2445111] and is subject to IEEE copyright. 


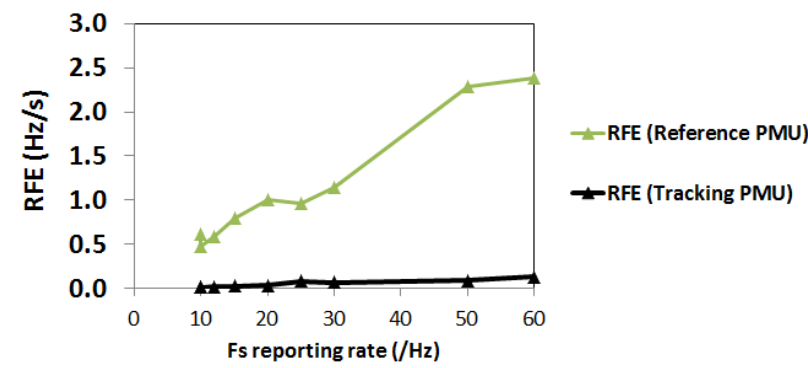

Fig. 16. Maximum Rate of Change of Frequency Error (RFE) results during OOB tests

\section{CONCLUSIONS}

The new standard C37.118.1a-2014 is a useful amendment to C37.118.1-2011. The Reference algorithm within C37.118.1a is now compliant, as well as PMUs from most (if not all) major PMU manufacturers. There are some areas where, from an application perspective, limits (particularly RFE) would ideally be tightened or additional scenarios tested, but this really requires a greater engagement between the PMU user community and the C37.118.1a standard committee, to agree on realistic conditions, evidence, expectations and limits.

Filter masks for both fixed-filter and frequency-tracking devices have been derived. The mask defining the beginning of the stopband needs to be up to $14.8 \%$ narrower for a frequencytracking PMU than for a fixed-filter PMU. This is partly due to the exact way that the present OOB test is conducted, and partly due to the inherent frequency-tracking property.

Implementing a good filter and algorithm requires a careful balance between, in particular, the bandwidth and OOB tests. These tests have both altered under C37.118.1a. Theoretical derivations in this paper, backed up with C37.118.1a tests in a simulated environment, show that the passband flatness of the main filter used to produce the phasor results can be relaxed to about $2.5 \mathrm{~dB}$ for a frequency-compensated or frequencytracking PMU. The reduced flatness can be traded off, in order to provide a faster cutoff to the stopband and a deeper broadband stopband rejection. Furthermore, it is possible to define different masks for the filter paths used to produce the phasor, frequency, and ROCOF outputs. The flatness of the frequency-path and ROCOF-path filters does not generally need to be as flat as $2.5 \mathrm{~dB}$. By further relaxing the flatness of filters used in these paths, wideband noise, harmonic and interharmonic rejection in the frequency and ROCOF measurement paths can be further improved.

\section{REFERENCES}

[1] IEEE, "IEEE Standard for Synchrophasor Measurements for Power Systems," C37.118.1-2011, 2011.

[2] IEEE, "IEEE Standard for Synchrophasor Measurements for Power Systems -- Amendment 1: Modification of Selected Performance Requirements " C37.118.1a-2014, 2014.

[3] National_Grid, "Frequency Changes during Large Disturbances and their Impact on the Total System," National Grid, Proposal for consultation, 15th August 2013. Available: http://www.nationalgrid.com/NR/rdonlyres/D3F18F81-BFE8-4BA18B82-CCD6CD0A0A4F/62018/GC0035IndustryConsultationv10.pdf, accessed Sep 2013.
[4] A. J. Roscoe, I. F. Abdulhadi, and G. M. Burt, "P and M Class Phasor Measurement Unit Algorithms using Adaptive Cascaded Filters," IEEE Transactions on Power Delivery, vol. 28, pp. 1447-1459, 2013.

[5] A. J. Roscoe, "Exploring the relative performance of frequency-tracking and fixed-filter Phasor Measurement Unit algorithms under C37.118 test procedures, the effects of interharmonics, and initial attempts at merging $\mathrm{P}$ class response with $\mathrm{M}$ class filtering," IEEE Transactions on Instrumentation and Measurement, vol. 62, pp. 2140-2153, 2013.

[6] D. Belega and D. Petri, "Performance of Synchrophasor Measurements Provided by the Weighted Least Squares Approach," in IEEE I2MTC 2013, Minneapolis, 2013, pp. 946-951.

[7] M. A. Platas-Garza and J. A. d. 1. O. Serna, "Dynamic Harmonic Analysis Through Taylor-Fourier Transform," IEEE Transactions on Instrumentation and Measurement, vol. 60, pp. 804-813, 2011.

[8] P. Castello, M. Lixia, C. Muscas, and P. A. Pegoraro, "Adaptive TaylorFourier synchrophasor estimation for fast response to changing conditions," 2012 IEEE International Instrumentation and Measurement Technology Conference (I2mtc), pp. 294-299, 2012.

[9] D. Belega and D. Petri, "Accuracy Analysis of the Multicycle Synchrophasor Estimator Provided by the Interpolated DFT Algorithm," IEEE Transactions on Instrumentation and Measurement, vol. 62, pp. 942-953, May 2013.

[10] P. Romano and M. Paolone, "Enhanced Interpolated-DFT for Synchrophasor Estimation in FPGAs: Theory, Implementation, and Validation of a PMU Prototype," IEEE Transactions on Instrumentation and Measurement, 2014.

[11] P. Castello, J. Liu, C. Muscas, P. A. Pegoraro, F. Ponci, et al., "A Fast and Accurate PMU Algorithm for P+M Class Measurement of Synchrophasor and Frequency," IEEE Transactions on Instrumentation and Measurement, vol. 63, pp. 2837 - 2845, 2014.

[12] J. A. de la O Serna, "Synchrophasor Estimation using Prony's Method," IEEE Transactions on Instrumentation and Measurement, vol. 62, pp. 2119-2128, 2013.

[13] I. Kamwa, S. R. Samantaray, and G. Joos, "Compliance Analysis of PMU Algorithms and Devices for Wide-Area Stabilizing Control of Large Power Systems," IEEE Transactions on Power Systems, vol. 28, pp. 1766-1778, 2013.

[14] I. Kamwa, S. R. Samantaray, and G. Joos, "Wide Frequency Range Adaptive Phasor and Frequency PMU Algorithms," IEEE Transactions on Smart Grid, vol. 5, 2014.

[15] A. J. Roscoe, "Frequency tracking, C37.118.1 a compliant, P and M class PMU (Phasor Measurement Unit) algorithm examples for MATLAB Simulink," 2014 Available: http://strathprints.strath.ac.uk/48245/, accessed Oct 2014.

[16] A. J. Roscoe, "Adaptive-window PMU algorithms using cascaded boxcar filters to meet and exceed C37.118.1(a) requirements," Workshop on Synchrophasor estimation processes for Phasor Measurement Units: algorithms and metrological characterisation, 2014. Available: http://desl-

pwrs.epfl.ch/files/content/sites/desl/files/Website/education/special/work shop-slides/S_2014_EMRP_PMU_Workshop_Adaptive_Boxcars.pdf, accessed Jan 2015.

[17] M. Bertocco, G. Frigo, C. Narduzzi, C. Muscas, and P. A. Pegoraro, "Compressive sensing plus Taylor-Fourier Transform for synchrophasor estimation," in Applied Measurements for Power Systems Proceedings (AMPS), Aachen, 2014.

[18] Arbiter Systems, "Model 1133A Phasor Measurement Specifications," 2012 Available: http://www.arbiter.com/files/productattachments/1133_phasor_measurement_specifications.pdf, accessed April 2012.

[19] National Institute of Standards and Technology (NIST), "Lessons learned from the NIST assessment of PMUs," 2014 Available: https://www.naspi.org/File.aspx?fileID=1250, accessed May 2014.

[20] K. E. Martin, A. R. Goldstein, M. G. Adamiak, G. Antonova, M. Begovic, et al., "Synchrophasor Measurements under the IEEE Standard C37.118.1-2011 with amendment C37.118.1a," IEEE Transactions on Power Delivery, 2015.

[21] IEEE, "IEEE Synchrophasor Measurement Test Suite Specification," ISBN 978-0-7381-9360-1, 2014.

This is a postprint of a paper submitted to and accepted for publication in IEEE Transactions on Instrumentation and Measurement [http://dx.doi.org/10.1109/TIM.2015.2445111] and is subject to IEEE copyright. 


\section{BIOGRAPHIES}

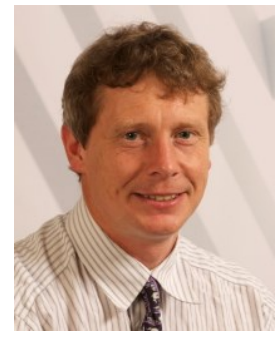

Andrew J. Roscoe received the B.A. and M.A degree in Electrical and Information Sciences Tripos at Pembroke College, Cambridge, England in 1991 \& 1994. Andrew worked for GEC Marconi from 1991 to 1995 , where he was involved in antenna design and calibration, specialising in millimetre wave systems and solid-state phasedarray radars. Andrew worked from 1995 to 2003 with Hewlett Packard and subsequently Agilent Technologies, in the field of microwave communication systems, specialising in the design of test and measurement systems for personal mobile and satellite communications. Since 2004, Andrew has been working at the University of Strathclyde in the field of power systems. His specialist areas include microgrid management, power system measurement algorithms, marine power systems, laboratory demonstration with power-hardware-in-the loop capability, and the integration of high penetrations of converter-connected renewables.

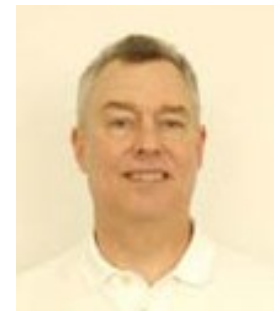

Bill Dickerson is Chairman of the Board of Arbiter Systems, Inc. in Paso Robles, California. Bill received his BSEE from Washington University in St. Louis in 1975 and his Masters in Business Administration from the University of Michigan in 1979. Bill worked at Hewlett-Packard Company in Palo Alto, California and Spokane, Washington from 1979 until 1986, when he and his current partners bought Arbiter Systems, Inc. Arbiter Systems is the industry leader in precise measurements for power systems and in accurate, reliable timing products for the power industry. Bill was the original product designer for Arbiter's GPS timing product line and the Model 1133A Power Sentinel, the industry's most accurate Phasor Measurement Unit (PMU).

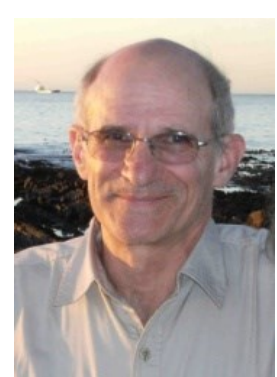

Kenneth Martin is a consulting engineer with the Electric Power Group (EPG). He has over 35 years experience in the electric utility industry, first at the Bonneville Power Administration (BPA) in communication, precise timing, instrumentation, and testing. He started working with synchrophasor measurement with the original PMUs in 1987 and conducted the first PMU tests. He developed the phasor measurement system at BPA including building the first phasor data concentrator, and supported similar developments at many utilities. Mr. Martin chaired the development of the IEEE C37.118 Synchrophasor Standards from the 2005 original, to the 2011 updates, and the 2014 amendment. He was a lead for the IEC 61850, part 905 and is the convener for 60255-118-1 developing the joint IEC-IEEE measurement standard. Mr. Martin is a Fellow of the IEEE and a registered Professional Engineer. He has authored or co-authored more than 60 articles and technical papers. loop capability, and the integration of high penetrations of converter-connected renewables.

This is a postprint of a paper submitted to and accepted for publication in IEEE Transactions on Instrumentation and Measurement [http://dx.doi.org/10.1109/TIM.2015.2445111] and is subject to IEEE copyright. 